\title{
Vertical and Lateral Delineation of Tar and Heavy Oil Reservoirs; Practical Considerations for a Challenging Task - Towards Optimum and Improved Recovery
}

\author{
Kamil Idris, Reservoir Characterization Department, Saudi Aramco
}

\begin{abstract}
Tar and heavy oils have imposed several challenges to the oil industry professionals in many aspects. Starting from definition, characterization, identification, delineation and through to development and production. Proper vertical and lateral delineation of tar and heavy oil reservoirs in a quantitative and a qualitative manner is imperative for proper reserves assessment, well placement and optimal field development plans.
\end{abstract}

Challenges in Vertical and lateral delineation of tar and heavy oil reservoirs include, but are not limited to;

1) Unavailability of unique indicators of tar/heavy oil. Most of the indicators rely on inference.

2) Gradation in fluid viscosity from tar to lighter oil and difficulty in quantifying each.

3) Variable form of occurrence of tar/heavy oil in the reservoirs as partial or total pore fillings or in a Solid Reservoir Bitumen (SRB) form.

4) Association with certain reservoir facies with certain petrophysical properties.

5) Major limitations of all tar identification tools and techniques,

6) Lack of clear understanding of paleo- and present structural controls in some areas.

In the face of these and many other challenges, the industry professionals have been trying to rely on many techniques, data, and tools to identify, describe and characterize tar and heavy oils. Such techniques, data, and tools include but are not limited to;

1) Core data is of course the most reliable hard data in visual tar/heavy oil identification.

2) Geochemical analyses including Pyrolitic Oil Productivity Index (POPI) of cuttings, core plugs and/or whole cores.

3) Recent advancements in logging and introduction of tools such as Nuclear Magnetic Resonance with its advanced interpretation and viscosity determination equations.

4) Multiple Pressure measurement and sampling tools and the recent capabilities of obtaining such data in horizontal wells.

5) Deviated and horizontal wells extending for kilometers provide essential opportunity to acquire and obtain most of the above mentioned hard and soft data. Advances in Geo-Steering techniques have made it possible to identify tar and heavy oil zones and place horizontal wells as desired whether producer and/or an injector.

"One hand can't clamp"; this saying may hold true if we try to use any single one of the above techniques or tools on its own to identify and characterize tar and heavy oil reservoirs. But proper integration of all available raw and interpreted data, each with its weighted level of confidence and uncertainty is the key to reach a sound tar/heavy oil distribution and characterization and the use of this for improving all aspects of development plans, reserves assessment, and well placement. 